Supporting Information

\title{
End-on Chain Orientation of Poly(3-alkyl thiophene)s on a Substrate by Microphase Separation of Lamellar forming Amphiphilic Diblock Copolymer
}

Kyu Seong Lee, ${ }^{+\dagger}$ Philgon Kim, ${ }^{+\dagger}$ Jaeyong Lee, ${ }^{\dagger}$ Chungryong Choi,${ }^{\dagger}$ Yeseong Seo,${ }^{\dagger}$ So Yeong Park, ${ }^{\dagger}$ Keonwoo Kim, ${ }^{\dagger}$ Chaneui Park, ${ }^{\ddagger}$ Kilwon Cho, ${ }^{*}$ Hong Chul Moon, ${ }^{\S}$ Jin Kon $\mathrm{Kim}^{* \dagger}$

†National Creative Research Initiative Center for Smart Block Copolymers, Department of Chemical Engineering, Pohang University of Science and Technology, Pohang, Kyungbuk 37673, Republic of Korea

\#Department of Chemical Engineering, Pohang University of Science and Technology, Pohang, Kyungbuk 37673, Republic of Korea $\S$ Department of Chemical Engineering, University of Seoul, Seoul 02504, Republic of Korea

* To whom correspondence should be addressed (jkkim@postech.ac.kr),

${ }^{+}$These authors contributed equally 


\section{Synthesis of P3DDT- $b$-P3TEGT copolymers}

\section{Synthesis of 3-(2-(2-(2-methoxyethoxy)ethoxy)ethoxy)methyl thiophene (2)}

2 was synthesized according to the previous reports. ${ }^{1,2}$ First, 3-bromomethylthiophene (15.0 g, $0.0847 \mathrm{~mol})$ (1) and triethylene glycol monomethyl ether (18.0 g, $0.110 \mathrm{~mol})$ were placed in 3-neck round-bottom flask (r.b.f.) in ice bath. Then, $250 \mathrm{~mL}$ of tetrahydrofurane (THF) was added under nitrogen purging. After $30 \mathrm{~min}, \mathrm{NaH}(8.33 \mathrm{~g})$ was added and the solution was stirred for $48 \mathrm{~h}$. The reaction was quenched by adding water and extracted with ether. Pure 2 was obtained by silica gel column chromatography using ethyl acetate:hexane $(3: 7)$ as an eluent.

\section{Synthesis of 2,5-dibromo-3-(2-(2-(2-methoxyethoxy)ethoxy)ethoxy)methyl thiophene} (TEGT monomer (3))

3 was synthesized by bromination of 2 using NBS. 2 was dissolved in $200 \mathrm{~mL}$ acetic acid/THF (1:1) under nitrogen purging. To the reaction mixture, NBS was added and the reaction was run at room temperature for $2 \mathrm{~h}$ with the light blocked. The reaction was quenched by adding water and extracted with chloroform. The chloroform solution was washed with $5 \%$ $\mathrm{KOH}$ solution. Pure $\mathbf{2}$ was obtained by silica gel column chromatography using ethyl acetate:hexane (3:7) as an eluent.

\section{Synthesis of P3TEGT (4) and P3DDT (5) homopolymers}

3 (2.0 g, $4.7 \mathrm{mmol}), t$-BuMgCl (1.0 M in THF, $4.7 \mathrm{~mL}, 4.7 \mathrm{mmol})$, anhydrous THF (5 mL) were introduced into a 2-neck r.b.f. under nitrogen purging. After $4 \mathrm{~h}$ mixing, anhydrous THF $(50 \mathrm{~mL})$ was additionally introduced. The polymerization was initiated with $\mathrm{Ni}(\mathrm{dppp}) \mathrm{Cl}_{2}$ and 
the mixture was stirred for $30 \mathrm{~min}$ at room temperature. About $5 \mathrm{~mL}$ of $\mathrm{HCl}(5 \mathrm{~N})$ solution was quickly injected to quench the reaction and extracted with water and chloroform. 4 was obtained by precipitation into excess cold n-hexane. 5 was synthesized by the same method as 4 except that 2,5-dibromo-3-dodecylthiophene was used as the monomer. After the polymerization of $\mathbf{5}$, reaction solution was also quenched using $\mathrm{HCl}$ solution and followed by precipitation into excess methanol.

\section{Synthesis of P3DDT-b-P3TEGTs (6)}

2,5-dibromo-3-dodecylthiophene (2.0 g, $4.8 \mathrm{mmol}), t$-BuMgCl (1.0 M in THF, $4.8 \mathrm{~mL}$, $4.8 \mathrm{mmol})$, anhydrous THF ( $5 \mathrm{~mL}$ ) were introduced into one of the 2-neck r.b.f. under nitrogen purging. At the same time, $4.7 \mathrm{mmol}$ of $\mathbf{3}$ was reacted with $t$ - $\mathrm{BuMgCl}$ in the other r.b.f.. After $4 \mathrm{~h}$ mixing, anhydrous THF $(50 \mathrm{~mL})$ was additionally introduced in 2,5-dibromo-3dodecylthiophene solution. The polymerization was initiated with $\mathrm{Ni}(\mathrm{dppp}) \mathrm{Cl}_{2}$. After stirring for $30 \mathrm{~min}$ at room temperature, solution $\mathbf{3}$ was transferred to the solution via a double-tipped needle, and the resulting solution was stirred for $1 \mathrm{~h}$. About $5 \mathrm{~mL}$ of $\mathrm{HCl}(5 \mathrm{~N})$ solution was quickly injected to quench the reaction, followed by precipitation into excess methanol. Synthesized block copolymers were purified by sequential column chromatography using nhexane, toluene, and THF as eluents. 
(a)



Figure S1. ${ }^{1} \mathrm{H}$ NMR of (a) 3-(2-(2-(2-methoxyethoxy)ethoxy)ethoxy)methyl thiophene, (b) TEGT monomer and (c) DT-48. The weight fraction of P3DDT was calculated by characteristic peaks of P3DDT and P3TEGT observed at $\delta 2.5$ - 2.8 ppm and $\delta 4.4$ - 4.8 ppm, respectively. 




Figure S2. SEC traces of P3DDT and P3TEGT homopolymers, DT-48, and DT-65. 

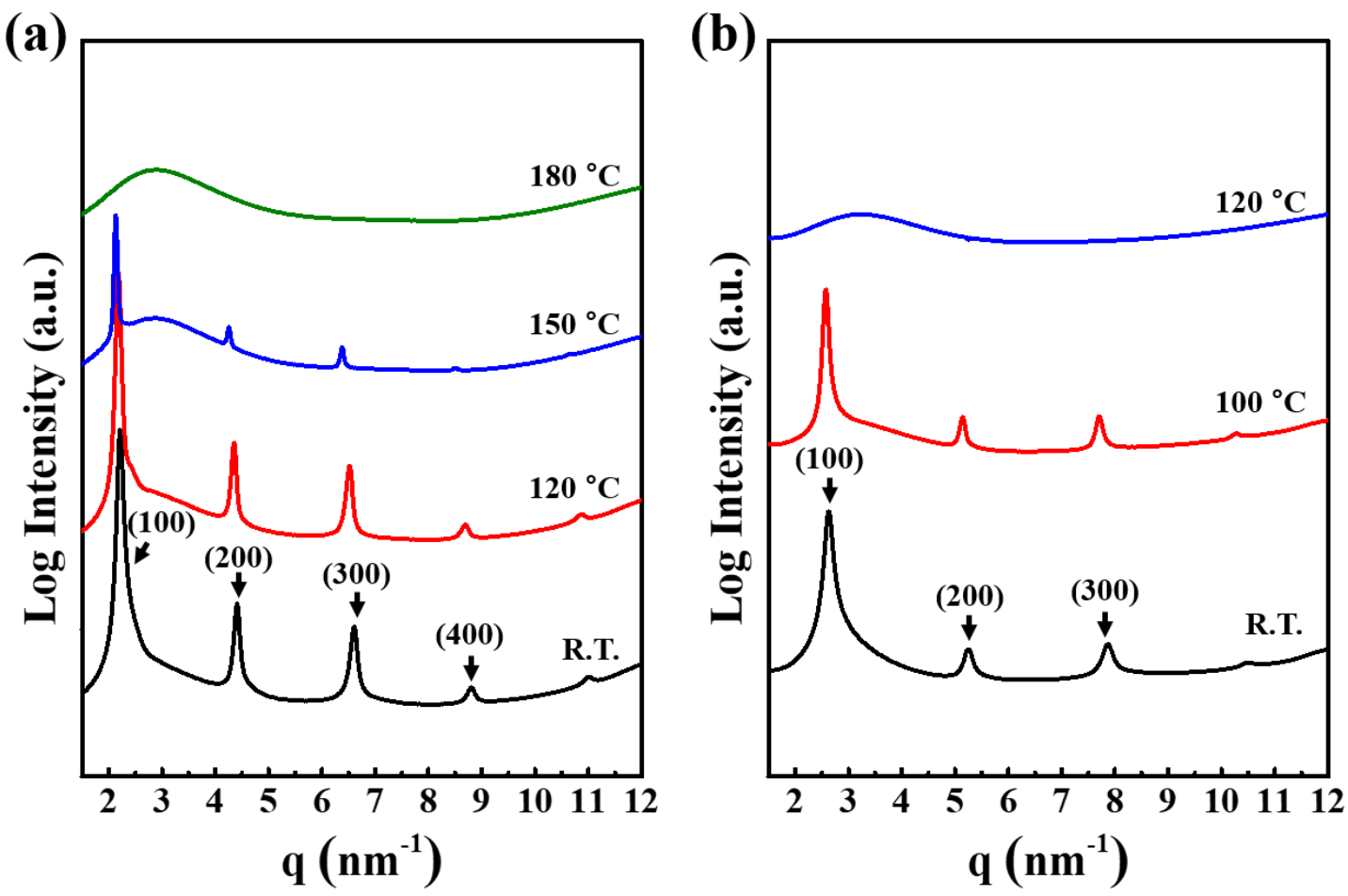

Figure S3. WAXS profiles for (a) P3DDT homopolymer and (b) P3TEGT homopolymer in bulk at various temperatures. 


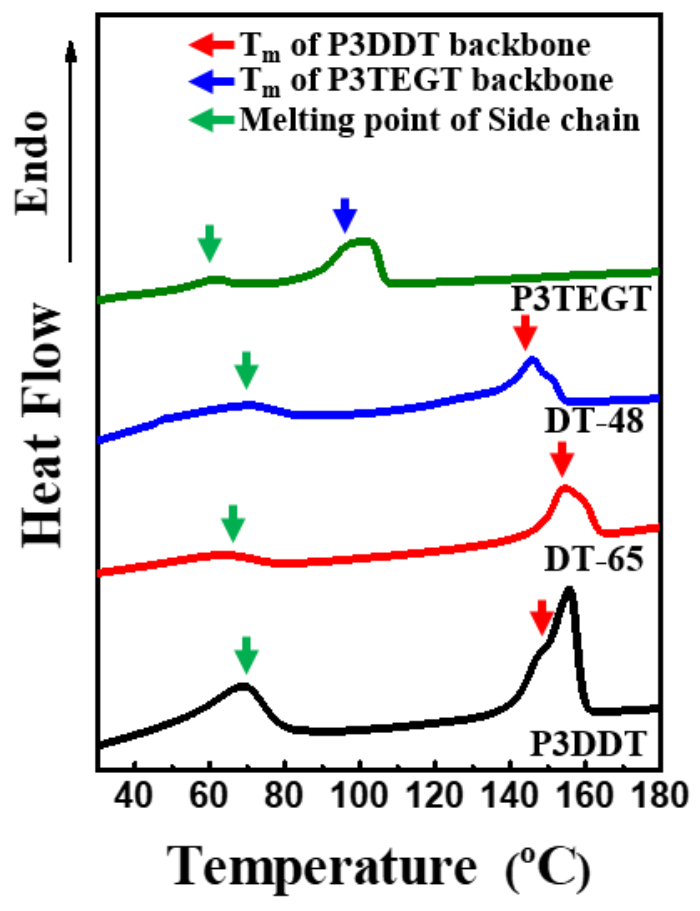

Figure S4. DSC thermograms obtained during the second heating run at a rate of $10^{\circ} \mathrm{C} / \mathrm{min}$ for P3DDT and P3TEGT homopolymers, DT-48, and DT-65. 

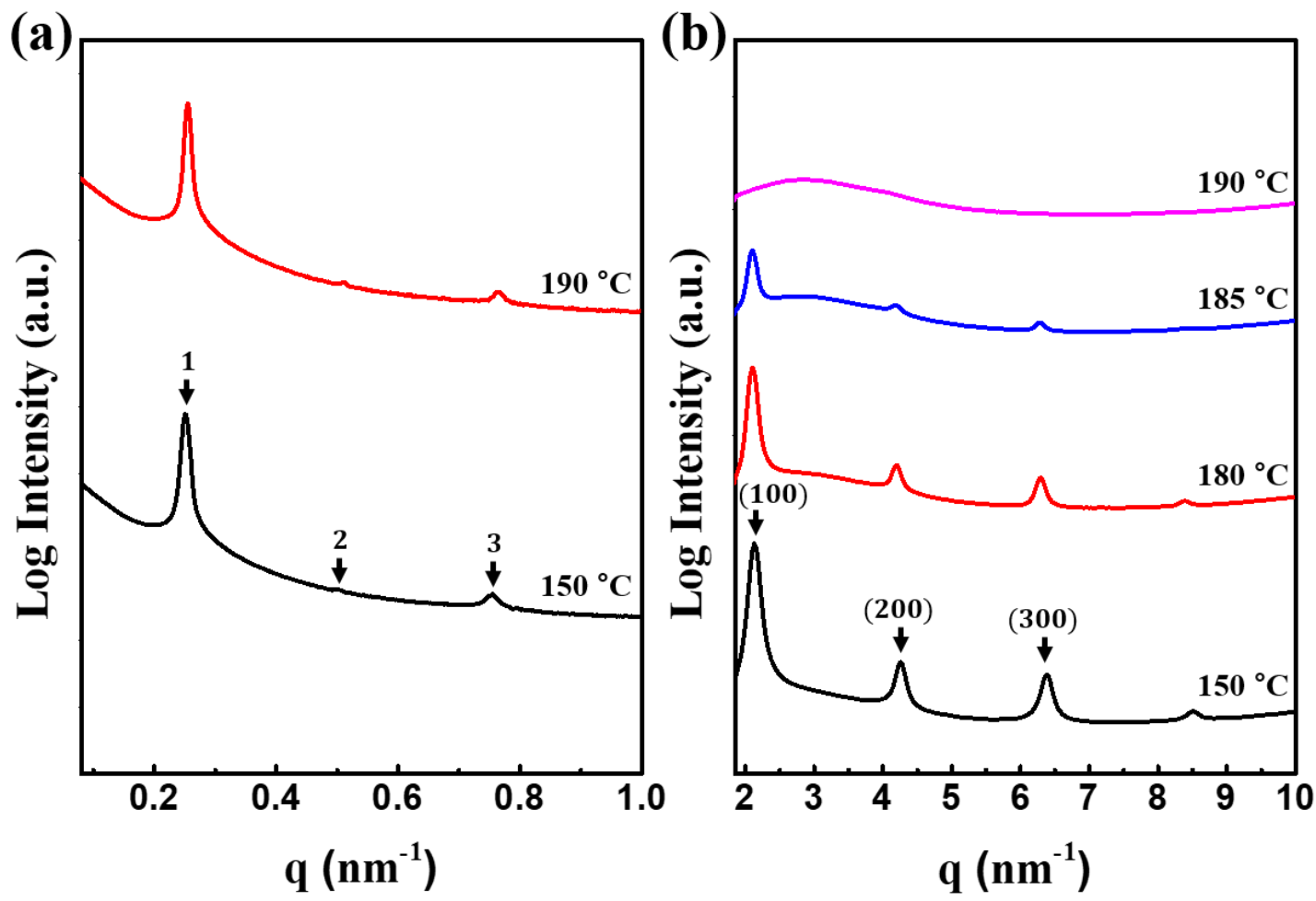

Figure S5. (a) SAXS and (b) WAXS profiles for DT-65 in bulk at various temperatures. 


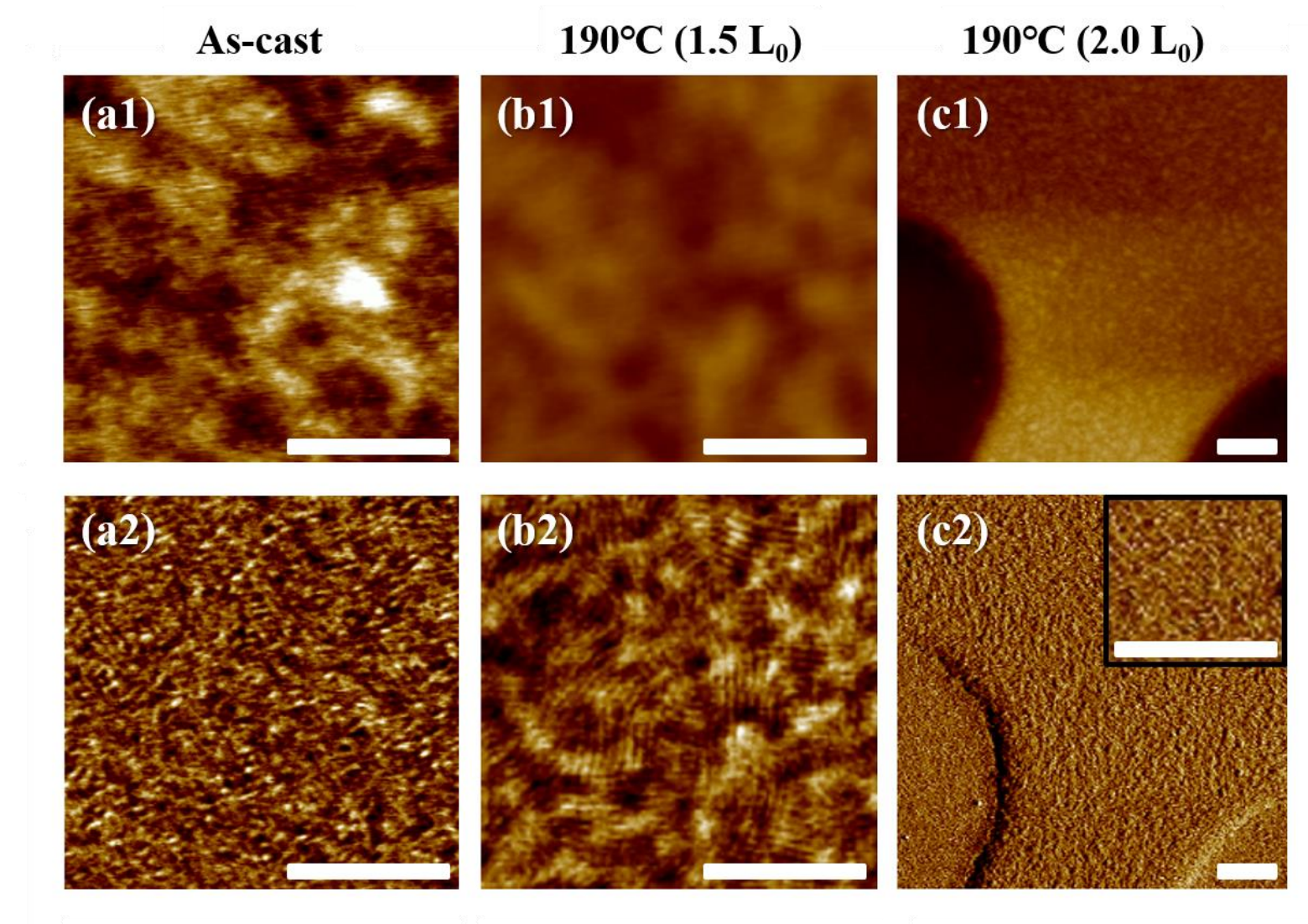

Figure S6. AFM height (upper panels) and phase contrast (lower panels) images of DT-65 at two different film thicknesses (a, b: $t=36.7 \mathrm{~nm}\left(1.5 \mathrm{~L}_{0}\right), \mathrm{c}: t=49.3 \mathrm{~nm}\left(2.0 \mathrm{~L}_{0}\right)$ before (a) and after thermal annealing at $190{ }^{\circ} \mathrm{C}$ for $1 \mathrm{~h}(\mathrm{~b}, \mathrm{c})$, followed by slow cooling to room temperature. The scale bar in all images is $400 \mathrm{~nm}$. 



Figure S7. XRR profiles for thermally annealed (a) DT-48 film ( $t=79.7 \mathrm{~nm})$ (b) DT65 film $(t=125.2 \mathrm{~nm})$ on a silicon substrate. The sample was annealed at $190{ }^{\circ} \mathrm{C}$, followed by slow cooling to room temperature.

Figure S7 shows XRR profiles of DT-48 and DT-65 thin film. The XRR profiles showed characteristic Bragg peaks at the postion of 1:2:3 relatives to the first order peaks $\left(\mathrm{q}^{*}=0.28 \mathrm{~nm}^{-1}, \mathrm{~L}_{0}=22.4 \mathrm{~nm}\right.$ for DT- $48, \mathrm{q}^{*}=0.265 \mathrm{~nm}^{-1}, \mathrm{~L}_{0}=23.7 \mathrm{~nm}$ for DT65), indicating that both DT thin films had lamellar structures with the lamellar plane parallel to the substrate. 

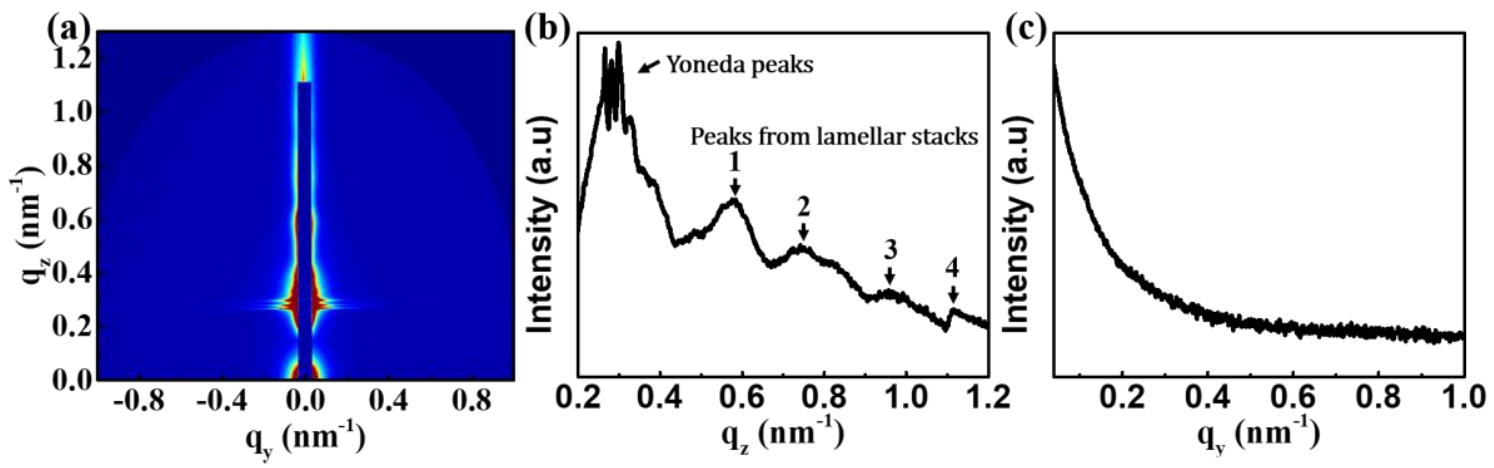

Figure S8. (a) GISAXS pattern, (b) out-of-plane near $\mathrm{q}_{\mathrm{y}}=0$ and (c) in-plane scans at $\mathrm{q}_{\mathrm{z}}=0.29 \mathrm{~nm}^{-1}$ of GISAXS of thermally annealed DT-65 thin film $(t=125.2 \mathrm{~nm})$. The sample was annealed at $190{ }^{\circ} \mathrm{C}$, followed by slow cooling to room temperature.

Figure S8 shows GISAXS results of thermally annealed DT-65 thin film. When the lamellar planes are parallel to the substrate, diffuse Bragg sheets (DBSs) appear along the out-of-plane direction (Figure S8b), in addition to no peak along the in-plane direction (Figure S8c) The Yoneda peaks ${ }^{3}$ of the P3DDT- $b$-P3TEGT thin film and the Si substrate were observed in the range of $0.266 \mathrm{~nm}^{-1}$ to $0.326 \mathrm{~nm}^{-1}$. 

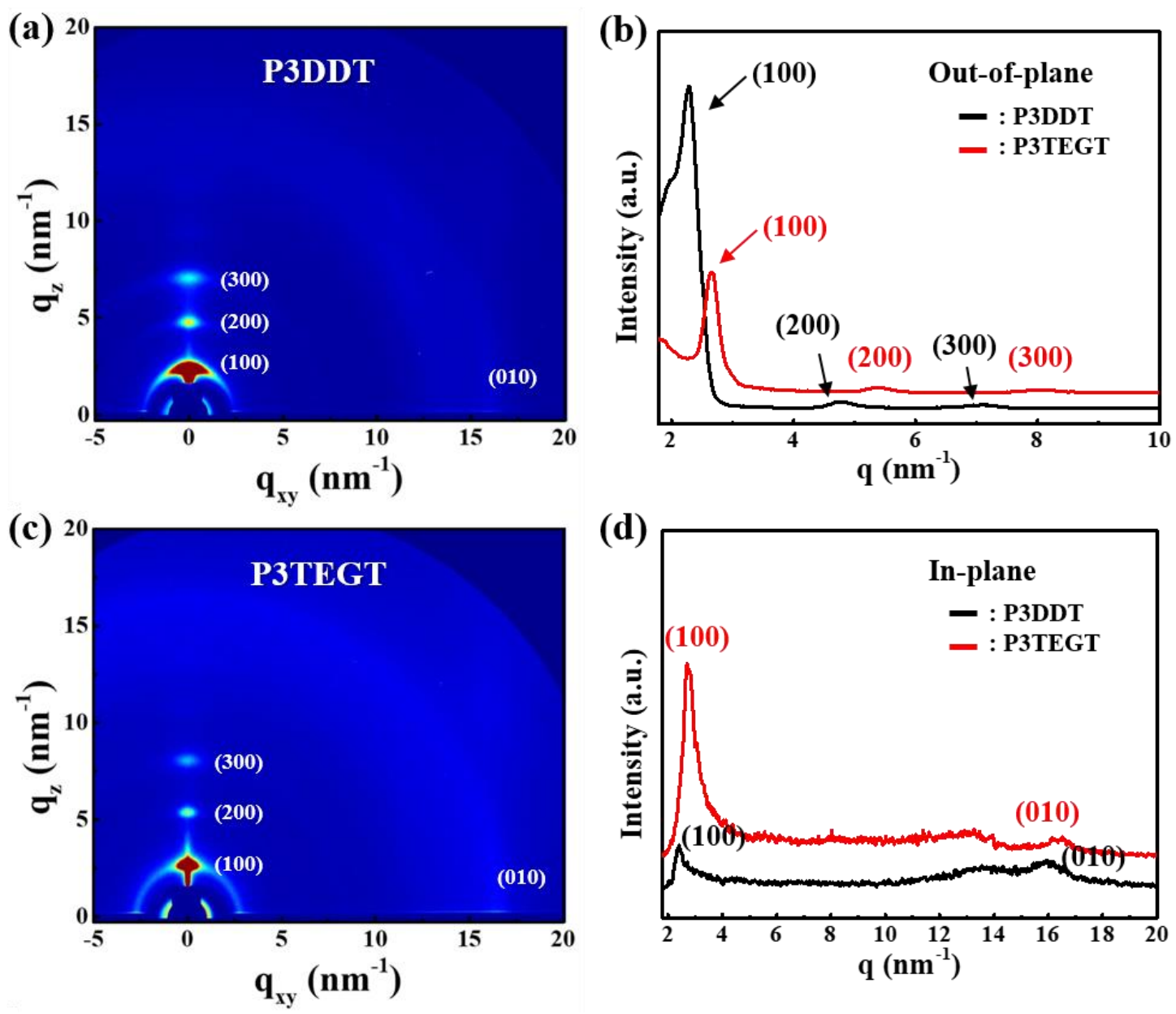

Figure S9. GIWAXS patterns for (a) P3DDT and (c) P3TEGT homopolymer on a pristine silicon wafer. (b) out-of-plane and (d) in-plane scans of GIWAXS. 
(a)
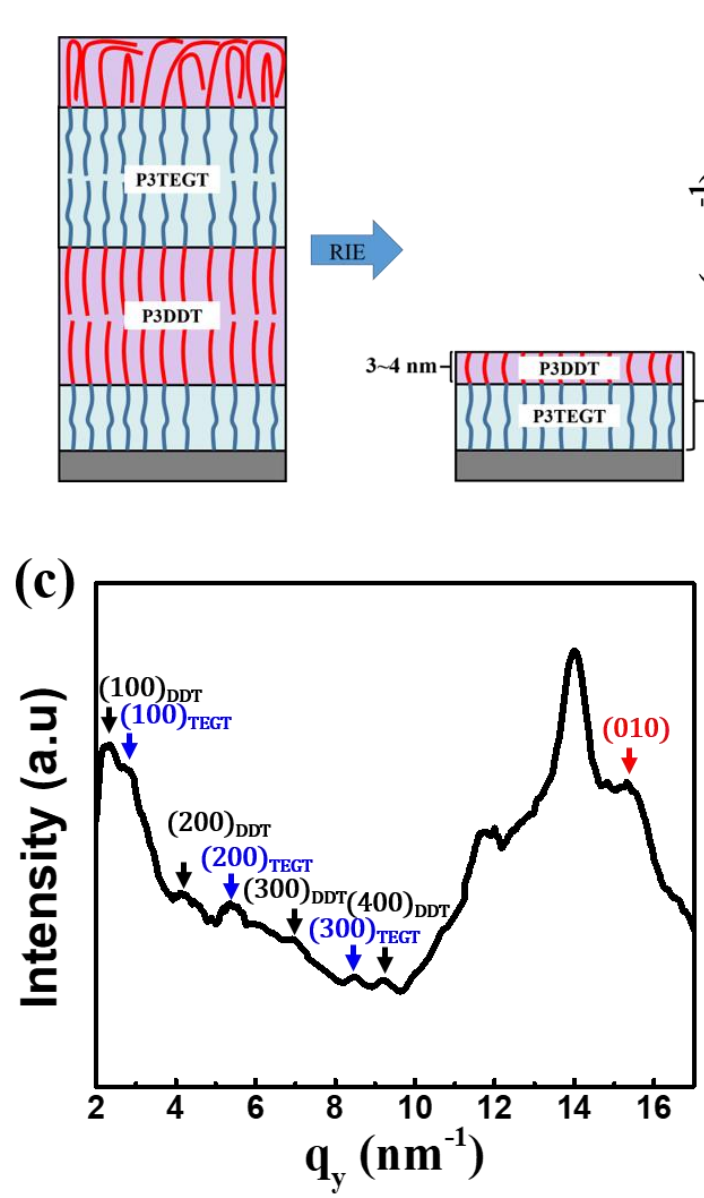
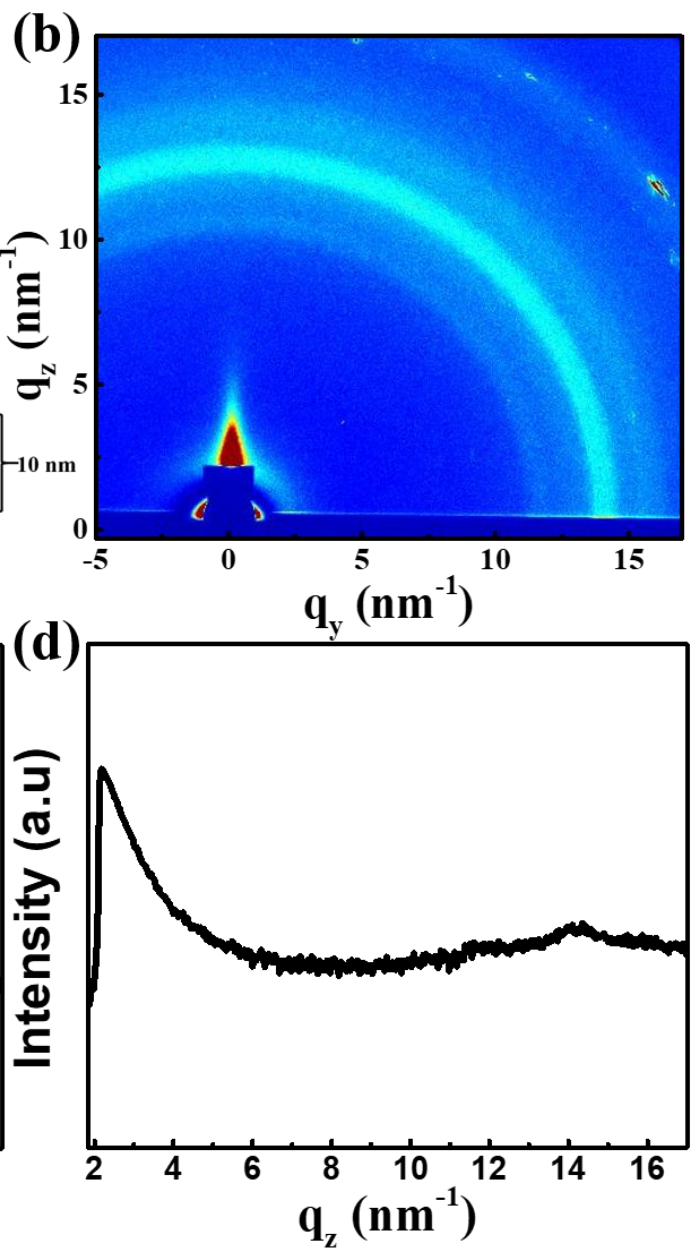

Figure S10. (a) Schematic of P3DDT and P3TEGT layers before and after RIE, (b) GIWAXS pattern, (c) in-plane and (d) out-of-plane scan of GIWAXS of thermally annealed DT-48 thin film after reactive-ion etching to remove most of P3DDT layers. The final thickness was 10 $\mathrm{nm}$. The sample was annealed at $190^{\circ} \mathrm{C}$, followed by slow cooling to room temperature.

Figure S10 shows GIWAXS results of thermally annealed DT-48 thin film after reactive-ion etching. After removing most of P3DDT layer, the final thickness of DT-48 thin film was 10 nm. In this case, one layer of P3TEGT $(6.5 \mathrm{~nm})$ including a small P3DDT microdomains (3.5 $\mathrm{nm}$ ) remain (Figure S10a). Figure S10c shows (100), (200), and (300) crystal peaks of P3DDT and P3TEGT chains along the in-plane direction. Those peaks indicated that P3DDT and 
P3TEGT chains had well-ordered end-on orientation. The GIWAXS peak at $q^{*}=12 \mathrm{~nm}^{-1}$ (domain spacing $=0.52 \mathrm{~nm}$ ) indicates the mean interdistance of the side groups between P3TEGT block chains, while another peak at $q^{*}=14 \mathrm{~nm}^{-1}$ (domain spacing $\left.=0.45 \mathrm{~nm}\right)$ represents the mean interdistance of the side groups between P3DDT block chains. ${ }^{1}$ Furthermore, crystal peaks corresponding to edge-on orientation was not observed along the out-of-plane direction (Figure S10d), indicating that DT-48 had only end-on orientation. 

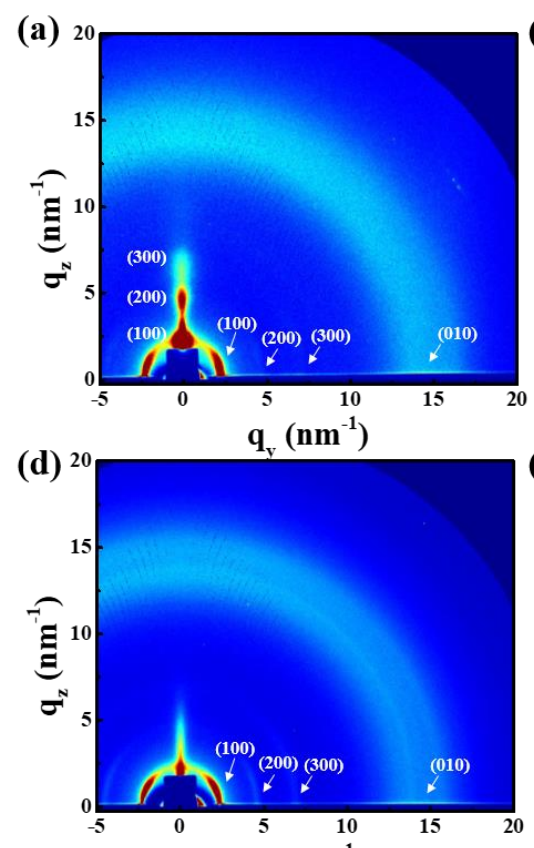

$\mathbf{q}_{\mathbf{y}}\left(\mathbf{n m}^{-1}\right)$

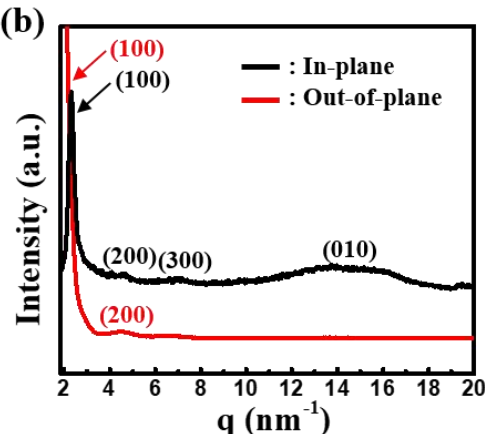

(c)
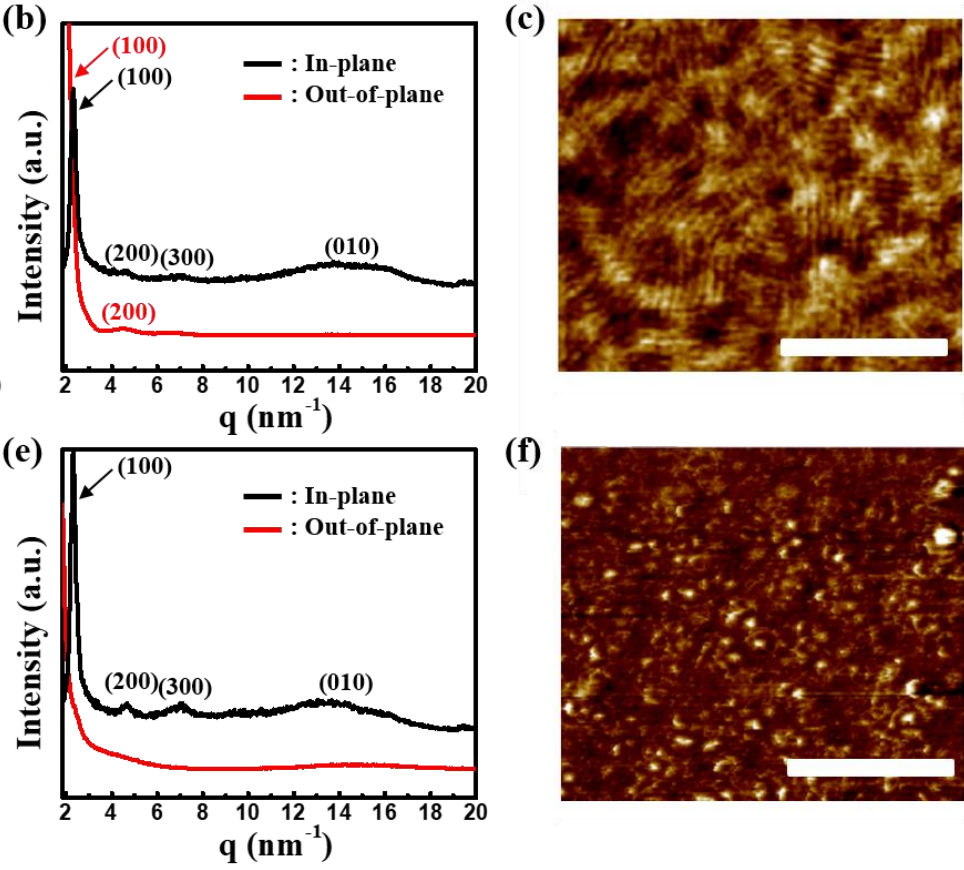

(f)

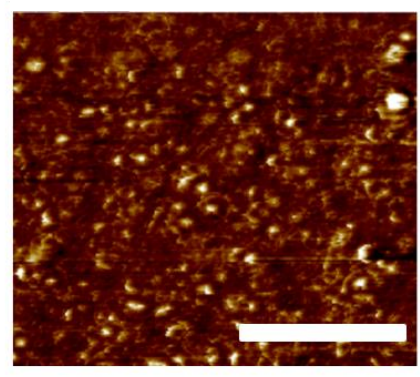

Figure S11. (a, d) GIWAXS patterns, (b, e) in-plane and out-of-plane scan of GIWAXS, (c, f) AFM phase contrast image of thermally annealed DT-65 thin film $(t=36.8 \mathrm{~nm}$ $\left(1.5 \mathrm{~L}_{0}\right)$. The sample was annealed at $190{ }^{\circ} \mathrm{C}$, followed by slow cooling to room temperature. The upper and lower panels represent before and after reactive-ion etching. To show more briefly, the intensity of in-plane scan in (b) is multiplied by a factor of 10. The scale bar in all images is $400 \mathrm{~nm}$. 


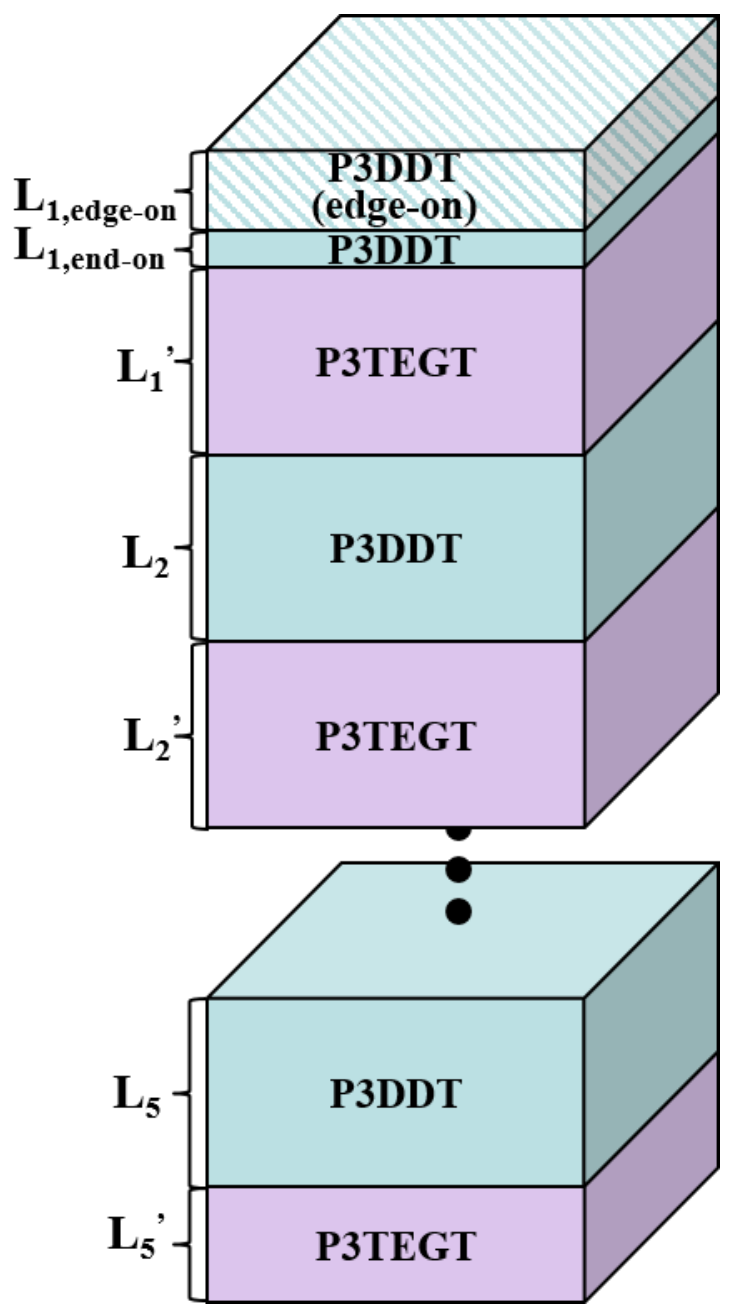

Figure S12. Schematic of P3DDT-b-P3TEGT with parallel oriented lamellar microdomains in a thin film with $100 \mathrm{~nm}\left(4.5 \mathrm{~L}_{0}\right)$.

Figure S12 shows P3DDT- $b$-P3TEGT chains with parallel oriented lamellar microdomains.

$$
\begin{gathered}
\mathrm{L}_{\mathrm{DDT}, \text { end-on }}=\mathrm{L}_{1, \text { end-on }}+\mathrm{L}_{2}+\cdots+\mathrm{L}_{5} \\
\mathrm{~L}_{\mathrm{DDT} \text {,edge-on }}=\mathrm{L}_{1, \text { edge-on }} \\
\mathrm{L}_{\mathrm{TEGT} \text {,end-on }}=\mathrm{L}_{1}^{\prime}+\mathrm{L}_{2}^{\prime}+\cdots+\mathrm{L}_{5}^{\prime}
\end{gathered}
$$

in which $\mathrm{L}$ is the thickness of the block copolymer layer. 


$$
\mathrm{L}_{\text {total }}=\mathrm{L}_{\mathrm{DDT} \text {,end-on }}+\mathrm{L}_{\mathrm{DDT} \text {,edge-on }}+\mathrm{L}_{\mathrm{TEGT} \text {,end-on }}
$$

According to the Matthiessen's rule, ${ }^{4}$

$$
\frac{1}{\mu_{\text {total }}}=\frac{1}{\mu_{\mathrm{DDT}, \text { end }- \text { on }}}+\frac{1}{\mu_{\mathrm{DDT} \text {,edge-on }}}+\frac{1}{\mu_{\mathrm{TEGT} \text {,end-on }}}
$$

in which $\mu$ is the hole mobility.

$$
\mathrm{R}_{\text {total }}=\mathrm{R}_{\mathrm{DDT} \text {,end-on }}+\mathrm{R}_{\mathrm{DDT} \text {,edge-on }}+\mathrm{R}_{\mathrm{TEGT} \text {,end-on }}
$$

in which $\mathrm{R}$ is the resistance.

$$
\mathrm{R}=\frac{\rho \mathrm{L}}{\mathrm{A}}=\frac{\mathrm{L}}{\mathrm{A} \sigma}
$$

in which $\rho$ is the resistivity, A is the area of top surface of P3DDT, and $\sigma$ is the conductivity.

$$
\begin{gathered}
\frac{\mathrm{L}_{\text {total }}}{A \sigma_{\text {total }}}=\frac{\mathrm{L}_{\mathrm{DDT}, \text { end }- \text { on }}}{A \sigma_{\mathrm{DDT}, \text { end }- \text { on }}}+\frac{\mathrm{L}_{\mathrm{DDT}, \text { edge-on }}}{A \sigma_{\mathrm{DDT}, \text { edge-on }}}+\frac{\mathrm{L}_{\mathrm{TEGT}, \text { end }- \text { on }}}{A \sigma_{\mathrm{TEGT}, \text { end-on }}} \\
\sigma=n_{i} q \mu
\end{gathered}
$$

in which $q$ is the electric charge $\left(1.6 \times 10^{-19} \mathrm{C}\right)$, and $n_{i}$ is the intrinsic current density. $n_{i}$ of P3DDT and P3TEGT layer is assumed to be same.

$$
n_{i, \text { total }} \approx n_{i, D D T} \approx n_{i, T E G T}
$$

The total hole mobility $\left(\mu_{\text {total }}\right)$ is extracted by the following equation:

$$
\frac{\mathrm{L}_{\text {total }}}{\mu_{\text {total }}}=\frac{\mathrm{L}_{\mathrm{DDT} \text {,end-on }}}{\mu_{\mathrm{DDT} \text {,end-on }}}+\frac{\mathrm{L}_{\mathrm{DDT} \text {,edge-on }}}{\mu_{\mathrm{DDT} \text {,edge-on }}}+\frac{\mathrm{L}_{\mathrm{TEGT}, \text { end }- \text { on }}}{\mu_{\mathrm{TEGT} \text {,end-on }}}
$$

Assuming that the edge-on orientation portions of DT-48 and DT-65 are $4 \%(4 \mathrm{~nm})$ at SCLC device, the estimated $\mu$ is summarized in Table $\mathrm{S} 1$. 
Table S1. Estimated hole mobilities of DT-48 and DT-65 at various values of Z.

$\begin{array}{ll}\text { Samplea,b } & \text { Estimated Hole mobility }\left(\mathbf{c m}^{\mathbf{2}} \mathbf{V}^{-\mathbf{1}} \mathbf{~ S}^{\mathbf{- 1}}\right) \\ \text { DT-48 }(Z=1) & 8.30 \times 10^{-7} \\ \text { DT-48 }(Z=10) & 6.93 \times 10^{-6} \\ \text { DT-48 }(Z=20) & 1.17 \times 10^{-5} \\ \text { DT-48 }(Z=30) & 1.52 \times 10^{-5} \\ \text { DT-48 }(Z=37) & 1.71 \times 10^{-5} \\ \text { DT-48 }(Z=40) & 1.79 \times 10^{-5} \\ \text { DT-48 }(Z=50) & 2.00 \times 10^{-5} \\ \text { DT-48 }(Z=100) & 2.62 \times 10^{-5} \\ \text { DT-65 }(Z=1) & 9.81 \times 10^{-7} \\ \text { DT-65 }(Z=10) & 7.96 \times 10^{-6} \\ \text { DT-65 }(Z=20) & 1.31 \times 10^{-5} \\ \text { DT-65 }(Z=30) & 1.66 \times 10^{-5} \\ \text { DT-65 }(Z=40) & 1.95 \times 10^{-5} \\ \text { DT-65 }(Z=50) & 2.16 \times 10^{-5} \\ \text { DT-65 }(Z=100) & 2.75 \times 10^{-5}\end{array}$

${ }^{\mathrm{a}} \mathrm{X}$ in DT-X indicates the weight percentage of P3DDT block. ${ }^{\mathrm{b}} \mathrm{Z}$ indicates the ratio of $\mu_{\text {end-on }}$ and $\mu_{\text {edge-on }}\left(\mu_{\text {end-on }} / \mu_{\text {edge-on }}\right)$.

From Table S1 and the measured hole mobility $\left(1.71 \times 10^{-5}\right.$ and $1.65 \times 10^{-5} \mathrm{~cm}^{2} \mathrm{~V}^{-1}$ $\mathrm{s}^{-1}$ ) of DT-48 and DT-65, the value of Z for DT-48 and DT-65 is estimated to be 37 and 30 , respectively. 

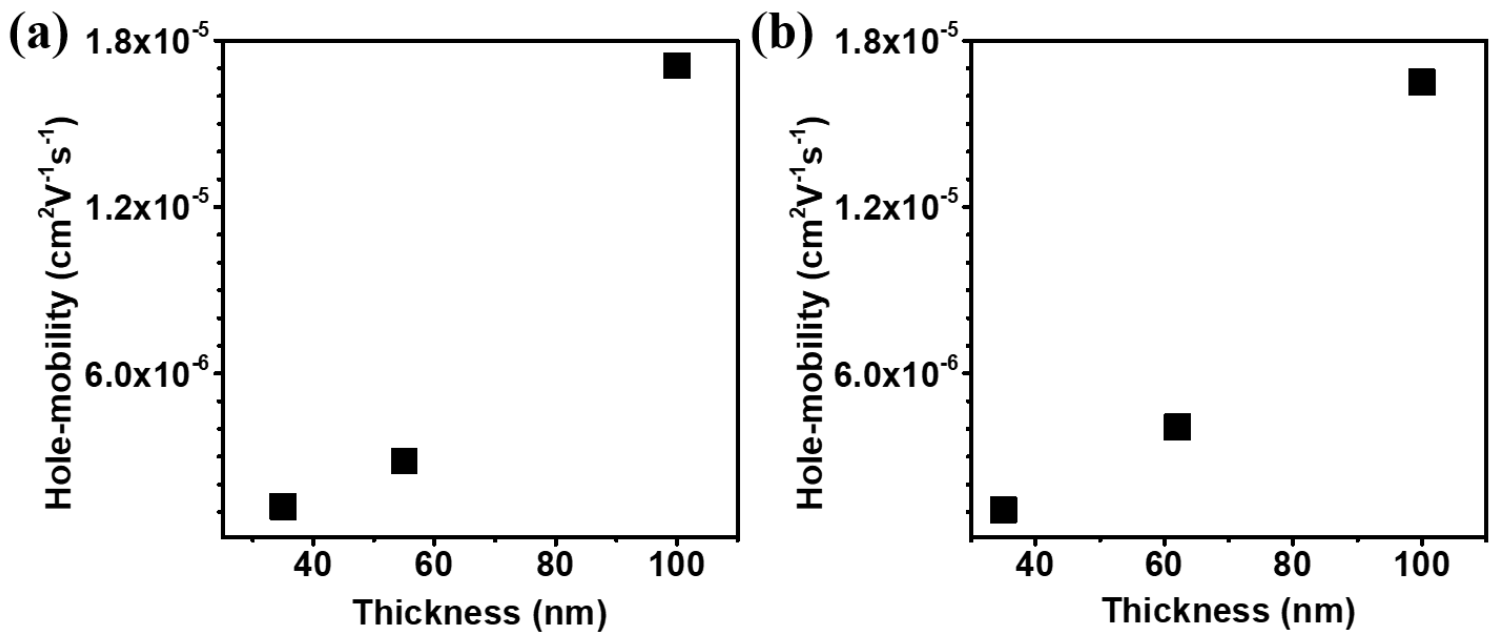

Figure S13. The hole-mobility of (a) DT-48 and (b) DT-65 with different thicknesses calculated from the J-V curves.

Figure S13 showed the hole-mobility $(\mu)$ of DT-48 and DT-65 with film different thicknesses. $\mu$ of DT with $\mathrm{t}<60 \mathrm{~nm}$ was relatively lower than that with $\mathrm{t}>100 \mathrm{~nm}$. This was because of a relatively large percent of fibril surface (>10\%) of thin film compared with that $(<4 \%)$ of thick film. At a large percent of fibril surface, the contribution of edge-on orientation to $\mu$ of DT became dominant. 


\section{References}

1. Higashihara, T.; Ohshimizu, K.; Ryo, Y.; Sakurai, T.; Takahashi, A.; Nojima, S.; Ree, M.; Ued, M. Synthesis and characterization of block copolythiophene with hexyl and triethylene glycol side chains. Polymer. 2011, 52, 3687-3695.

2. Shao, M.; He, Y. J.; Hong, K. L.; Rouleau, C. M.; Geohegan, D. B.; Xiao, K. A watersoluble polythiophene for organic field-effect transistors. Polym. Chem. 2013, 4, 5270-5274.

3. Sepe, A.; Zhang, J.; Perlich, J.; Smilgies, D.; Posselt, D.; Papadakis, C. M. Toward an equilibrium structure in lamellar diblock copolymer thin films using solvent vapor annealing An in-situ time-resolved GISAXS study. European. Polymer. Journal. 2016, 81, 607-620.

4. Bass, J. Deviations from Matthiessen's Rule. Adv. Phys. 1972, 21, 431-604 